\title{
Le recueil contre la revue
}

«Le problématique voyageur»

Marie-Ève Thérenty

\section{(2) OpenEdition}

\section{Journals}

Édition électronique

URL : http://journals.openedition.org/recherchestravaux/175

DOI : 10.4000/recherchestravaux.175

ISSN : 1969-6434

Éditeur

UGA Éditions/Université Grenoble Alpes

Édition imprimée

Date de publication : 15 avril 2007

Pagination : 29-38

ISBN : 978-2-84310-107-7

ISSN : 0151-1874

Référence électronique

Marie-Ève Thérenty, «Le recueil contre la revue », Recherches \& Travaux [En ligne], 70 | 2007, mis en ligne le 27 novembre 2008, consulté le 08 septembre 2020. URL : http://journals.openedition.org/ recherchestravaux/175; DOI : https://doi.org/10.4000/recherchestravaux.175 
Marie-Ėve THÉRENTY

Université Paul-Valéry-Montpellier 3-CERD

\section{Le recueil contre la revue «Le problématique voyageur»}

Le recueil a souvent été considéré au XIX siècle comme un mode de publication et de réédition commode pour des textes brefs qui ne peuvent à eux seuls former la matière d'un livre. Recueils d'articles journalistiques, keepsakes, albums témoignent de la vogue du recueil sous la monarchie de Juillet, tout comme un certain nombre d'appellations courantes renseignent sur la poétique compilatoire souvent associée à cette pratique : mélanges, salmigondis, macédoine, mosaïque... George Sand, très soucieuse de l'exploitation éditoriale de ses œuvres, n'a pas renié cette pratique et beaucoup de ces recueils "anthumes" " et plus encore posthumes ressemblent à des compilations : Souvenirs et impressions littéraires chez Dentu en 1862, Autour de la table d'abord chez Dentu en 1862 puis chez Lévy en I873, Impressions et souvenirs en I873, Dernières pages en I877, Nouvelles lettres d'un voyageur en I877, Questions d'art et de littérature en 1878 , Questions politiques et sociales en I879, Souvenirs de I848 en I880, Souvenirs et idées en I904. Dans sa préface de I843 aux Lettres d'un voyageur, George Sand rattache l'œuvre à cet usage de la compilation. Le geste fort du recueil commence donc par une dénégation de l'œuvre : "Jamais ouvrage, si ouvrage il y a, n’a été moins raisonné et moins travaillé que ces deux volumes de lettres écrites à des époques assez éloignées les unes des autres $[\ldots] »\left(\right.$ p. $645 ;$ p. $\left.37^{2}\right)$. Pourtant nous allons tenter de prouver que les Lettres d'un voyageur se détachent absolument de la pratique compilatoire et que George Sand, avec ce coup d'essai, a exploité en précurseur les possibilités esthétiques et idéologiques offertes par la forme du recueil.

I. Nous utilisons à dessein ce néologisme cher à Alphonse Allais.

2. Selon la convention que nous adoptons pour ce volume: G. Sand, Lettres d'un voyageur, pages de l'édition de G. Lubin puis pages de l'édition de H. Bonnet. 
Une des particularités des «Lettres d'un voyageur» est qu'elles ont été largement prépubliées dans une autre forme de recueil, la revue, avant d'être réunies dans un livre. Il nous semble d'abord intéressant de montrer que l'œuvre Lettres d'un voyageur, telle que nous la lisons aujourd'hui, n'existe pas avant I837 mais qu'il existe des textes épars susceptibles d'une certaine lecture que George Sand volontairement efface avec le geste fort du recueil. Le «recueilleur» se fait alors auteur car si la transposition efface nombre d'effets de sens produits par l'interaction avec l'actualité ou avec le "moment" de l'auteur, elle en produit d'autres par le montage qu'opèrent à la fois la mise en volume et la lecture en série. L'effet de collection des énonciations et des identités, souligné dans la préface, se complexifie notamment par le transfert des textes de la revue au recueil, faisant de cette étape et de ce passage la vraie genèse et la fondation de l'œuvre.

\section{L'effet revue}

À une exception près 3 , toutes les «Lettres d'un voyageur » sont donc parues en livraison dans la Revue des Deux Mondes entre le I5 mai I834 et le I5 novembre I836. Rappelons qu'il s'agit de la plus prestigieuse revue de la monarchie de Juillet : dirigée par Buloz, elle détient déjà une sorte de magistère intellectuel sur la société française. Les "Lettres d'un voyageur » y rencontrent immédiatement un certain succès, voire, en I836, un succès de scandale : mais est-ce à dire que les lecteurs de la revue ont lu la même œuvre que ceux qui lurent le recueil en 1837 chez Bonnaire et surtout que ceux qui lurent les Lettres avec préface en 1843 chez Perrotin ? Il me paraît nécessaire dans un premier temps de revenir aux lettres dans la revue pour tenter de comprendre - bien que l'expérience soit manifestement utopique et approximative - quelle pouvait être la lecture entre I834 et I836 des «Lettres d'un voyageur».

En fait, structurellement et poétiquement, le geste de la revue differe fondamentalement de celui du recueil. Les textes de la revue, dus à des auteurs multiples, sont réunis par le directeur de la publication (en l'occurrence ici Buloz) et correspondent à sa conception de l'actualité. Chaque lettre, au moment de sa parution, a donc vu sa lecture conditionnée par un co-texte et un contexte qui échappaient largement au contrôle de Sand.

Première information à tirer de cette immersion dans la revue : George Sand dans les années de parution des «Lettres d'un voyageur" est manifestement l'auteur phare, l'emblème de la revue. À plusieurs titres, son statut y est

3. Il s'agit de la "Lettre à M. Nisard" parue dans la Revue de Paris du 29 mai 1836. 
exceptionnel. Non seulement elle est la seule femme qui écrive régulièrement dans ce périodique qui ostracise complètement la femme auteur (durant toute la monarchie de Juillet, seules deux femmes auront l'honneur de "penser» dans la revue : George Sand jusqu'en I84I et Daniel Stern pour deux livraisons en 1844), mais en plus pratiquement chaque numéro publie du George Sand. Dès la fin I832, Buloz s'est attaché Sand par un contrat exclusif et elle est tenue de fournir chaque mois une feuille et demie à deux feuilles de copie. Entre I834 et I836, outre les «Lettres d'un voyageur", la revue publie également trois romans complets de Sand : Leone Leoni, André, Simon; et même les rares livraisons qui ne la publient sont hantées par le discours à son sujet (ainsi le numéro du ${ }^{\text {er }}$ octobre I834 comporte une critique de Gustave Planche sur Jacques et une fiction de Loève-Veimars, "Souvenirs de Normandie", mettant en scène un personnage féminin, Thécla, qui envoie des lettres d'amour recopiées dans un roman épistolaire de Sand). La revue construit donc une sorte de monument d'hommage à George Sand. Dans ce contexte, les "Lettres d'un voyageur", par leur forme épistolaire et leur écriture à la première personne, sont manifestement et massivement lues par les contemporains et pas forcément à contresens - car c'est aussi la stratégie de l'auteur - comme un texte autobiographique, voire d'autopromotion.

En outre, le caractère novateur du texte, en terme de poétique, est passé d'autant plus inaperçu que l'horizon d'attente du public était structuré par les formes de prédilection de la revue, soit le récit de voyage - et notamment le récit de voyage dans les Alpes ${ }^{4}$ et la correspondance de voyageur. Ainsi un grand texte paraît significativement en alternance, voire en concurrence avec les premières "Lettres d'un voyageur", il s'agit des Impressions de voyages de Dumas, récit de voyage classique et spirituel à grand succès publié également dans les années I833, I834 et I836. Les lettres s'insèrent dans un co-texte où les trois premières, notamment, ne semblent absolument pas détonner, ce qui sans doute a pu nuire à une lecture véritablement attentive de ces textes novateurs.

La lecture en contexte donne un autre sens aux textes car les «Lettres d'un voyageur", comme tout texte de revue, sont parsemées de références à l'actualité. En témoigne par exemple l'allusion à l'attentat de Fieschis dans la

4. Sur ce point, nous renvoyons le lecteur à Cl. Lacoste-Veysseyre, Les Alpes romantiques, le thème des Alpes dans la littérature française de I800 à I850, Genève, Slatkine, I98I.

5. "L'explosion d'une machine infernale, dont les résultats ont été bien assez funestes par eux-mêmes, a donné au despotisme de prétendus droits sur les plus purs ou sur les plus paisibles d'entre nos frères. On s'attend à des actes féroces de ce pouvoir insolent qui s'intitule l'ordre et la justice» (p. 849 ; p. 229). 
lettre sur Lavater, audacieuse dans le contexte de la revue de I835, pratiquement inaudible dans le recueil. Le prouve également la lettre du is septembre I834 sur Lamennais et sur son Paroles d'un croyant, qui s'insère dans un dialogue entre la Revue des Deux Mondes et Lamennais illustré par l'article du I ${ }^{\text {er }}$ mai 1834 de Sainte-Beuve sur Paroles d'un croyant. Significativement, lors du passage au recueil, George Sand supprime d'ailleurs dans la bouche de Liszt, à la lettre $\mathrm{X}$, une longue digression sur les Paroles d'un croyant comme si elle avait conscience de la péremption de l'actualité. Toute une série de coupes est destinée à supprimer des fragments d'actualité dépassée lors du passage au recueil. Le destinataire premier des «Lettres d'un voyageur» est donc bien le lecteur de la Revue des Deux Mondes pour un débat tout d'actualité qui, par la suite, deviendra beaucoup moins audible dans les hasards de l'histoire d'une relecture. Ainsi la lettre III prend un sens différent dans le recueil parce que George Sand a alors un passé avec Lamennais, qu'elle est l'auteur par exemple des Lettres à Marcie dans Le Monde en I837 et que sa connaissance de l'abbé n'est plus alors un "mensonge effronté» mais qu'au contraire, pour le lecteur, l'actualisation de son déni des relations avec Lamennais engage les Lettres dans la reconstruction fictionnelle.

À côté de cette stratégie de l'actualité, il existe également chez Sand ce qu'on pourrait appeler une stratégie du moment. L'écriture du moment est une écriture autobiographique de son actualité. C'est un terme qu'elle utilise souvent dans ses lettres comme dans celle qu'elle écrit à François Buloz le i7 mai 1836 :

Mon cher Buloz, je suis très en colère contre vous, de ce que vous n'avez pas mis mon article dans votre revue. Je tenais beaucoup à ce qu'il parût dans ce moment-ci, où je suis accusée par les sots de mille turpitudes, auxquelles ces lettres sont un démenti loyal et sincère. [...] Le gain de mon procès à Bourges dépend de l'impression du moment.

Participent à cette stratégie du moment toutes les "Lettres d'un voyageur» écrites au féminin dans la revue au moment du procès contre Casimir Dudevant. Cette écriture du moment est d'ailleurs l'objet d'une polémique à l'intérieur de la revue où Buloz et Didier sont contraints de commenter la nouvelle posture d'énonciation autobiographique sandienne. Didier écrit ainsi dans la livraison du I5 juin I836 pour défendre l'insertion de quelques lettres jugées trop autobiographiques par le public (lettres IV et IX) :

On sait que George Sand vient de gagner un procès grave et délicat. C'est à cette circonstance, capitale dans sa vie, que se rapportent quelques fragments de lettres insérés dans la livraison du $\mathrm{I}^{\mathrm{er}}$ juin. Ces révélations toutes personnelles ne paraissaient pas destinées à une publicité si prompte ; détachées d'un corps de Mémoires tout à fait individuels, elles ne devaient voir le jour que beaucoup plus tard. Si l'auteur s'est décidé à lever dès à présent le voile de sa vie privée et intime, 
c'est que les attaques violentes dirigées contre lui ont été le frapper jusque dans cet asile. Poussé dans ses derniers retranchements, le poète en use à sa manière ; il fait servir à sa défense les sentiments et les facultés que Dieu a mis en lui. Qui oserait l'en blâmer, surtout en présence des diatribes mensongères et niaises qu'on vient tout récemment de diriger contre lui.

On le voit ici, ces lettres lues dans le contexte de la revue annoncent à la fois très tôt le projet d'Histoire de ma vie mais aussi soulignent la naissance de ce que j'appelle le "journalisme intime» de George Sand, forme qui perdure et à laquelle participe notamment «Impressions et souvenirs» dans Le Temps à partir de i87I.

Il faut enfin souligner qu'entre le lecteur de revue et celui du recueil, la gestion du temps de la lecture differe sensiblement. Les articles dans la revue paraissent à des dates aléatoires entre mai I834 et novembre I836, et même s'il semble admis que les lecteurs de la revue étaient manifestement attentifs, il reste à réfléchir sur cette lecture intermittente qui gomme beaucoup plus la continuité qu'elle ne la souligne, qui crée d'autres effets de surprise et d'attente que la lecture de recueil. Publier par fragments, c'est accomplir un travail partiellement contradictoire avec l'opération de lecture qui est aussi opération de ligature. Dans la revue, peuvent ainsi apparaitre des effets de suspens qui disparaissent dans le recueil. À la fin de la première lettre, George Sand annonce un prochain voyage au Tyrol et donc sans doute une imminente narration. Alors que le lecteur de revue reste longuement dans l'attente finalement frustrée de ce récit, le lecteur du recueil sait, dès la page tournée, que cette annonce était un leurre. George Sand se contraint donc dans la revue à une pratique feuilletonesque de la narration avec ses effets de rappel nécessaires ${ }^{6}$, effets qui perdurent dans le recueil comme une trace de la lecture originelle puisqu'ils renvoient à la parution intermittente des lettres plus qu'à la possibilité induite par la forme du recueil et peut-être souhaitée par l'auteur d'une lecture en continu.

\section{L'effet recueil}

La lecture continue n'est rendue possible que par le recueil. Le fait est que la réunion de ces textes à la genèse disparate, à l'origine variée aboutit non pas à l'agrégation forcée de textes autonomes mais compose une totalité signi-

6. «En expiation de ces cérémonies païennes et républicaines, ils furent mis tous trois en prison avec une vingtaine d'assistants ; je crois t'avoir raconté cela dans une de mes lettres» (lettre III ; p. 73I ; p. II8). 
fiante. Cette œuvre composite est unifiée à la fois par la volonté "auctoriale" qui accomplit des gestes forts sur les textes mais aussi par la lecture. Car le recueil, comme en témoignent les recherches de poétique les plus récentes ${ }^{7}$, n'est pas seulement un support éditorial. L'unité du livre vient à la fois du contrat de lecture implicite au genre et également des effets de récurrences thématiques, structurelles et symboliques mis en évidence et en correspondance par le lecteur. La lecture des Lettres d'un voyageur repose sur une lecture active et donc sur la mise à jour de l'intratextualité, c'est-à-dire de l'ensemble des relations internes qui peuvent être établies entre les différentes lettres. La conscience de cette importance de la lecture est d'ailleurs soulignée par Sand dans sa préface puisqu'elle fait allusion au lecteur qu'elle désigne par cette périphrase : «le témoin inconnu »- expression curieuse et d'autant plus significative qu'elle rappelle le titre d'une de ses nouvelles récentes, "Le dieu inconnu».

Le tout n'est donc pas la seule somme de ces parties, car s'ajoute un supplément de sens qui ne s'inscrit pourtant en aucun lieu textuel. Ce supplément est autant voulu par l'auteur qu'accompli par le lecteur, lequel est véritablement en charge de la construction de la continuité. Ainsi le lecteur peut être sensible, par exemple, à l'insistance de la métaphore musicale et à la présence de plus en plus marquée de destinataires musiciens dans les lettres. Il pourra noter que, dès les premières lettres, George Sand fait l'éloge d'une poétique du décousu musical qui est aussi une mise en abyme de la poétique du recueil :

Rien n'embarrasse ces improvisateurs de pots-pourris. Une cavatine de Bellini devient sur le champ un chœur à quatre parties. Un chœur de Rossini s'adapte à deux voix au milieu d'un duo de Mercadante, et le refrain d'une vieille barcarolle d'un maestro inconnu, ralentie jusqu'à la mesure grave du chant d'église, termine tranquillement le thème tronqué d'un cantique de Marcello. Mais l'instinct musical de ce peuple sait tirer parti de tant de monstruosités, le plus heureusement possible, et lier les fragments de cette mutilation avec une hardiesse qui rend souvent la transition difficile à apercevoir. [...] Ignorants de la musique écrite, ces dilettanti passionnés vont recueillant dans leur mémoire les bribes d'harmonie qu'ils peuvent saisir à la porte des théâtres ou sous le balcon des palais. Ils les cousent à d'autres portions éparses qu'ils possèdent d'ailleurs, et les plus exercés, ceux qui conservent les traditions du chant à plusieurs parties, règlent la mesure de l'ensemble. (P. 697 ; p. 85.)

7. Voir par exemple : I. Langlet, Le Recueil littéraire, Pratique et théorie d'une forme, Rennes, Presses universitaires de Rennes "Interférences ", 2003 ; Études littéraires, 1998, vol. XXX ; F. Dumont, La Pensée composée. Formes du recueil et constitution de l'essai québecois, Québec, Éditions Nota bene "Les cahiers du Creliq ", I999; R. Audet, Des textes à l'ouvre. La lecture du recueil de nouvelles, Québec, Éditions Nota bene "Études», 2000 ; D. Alexandre, M. Frédéric et J.-M. Gleize, «Le Recueil poétique », Méthode!, nº 2, 2002. 


\section{Du recueil à la revue : le passage essentiel}

Il me semble cependant qu'opposer de manière binaire ces lectures, les "Lettres d'un voyageur» dans la revue et les Lettres d'un voyageur dans le recueil, l'effet revue et l'effet recueil, manquerait l'essentiel qui est le voyage du texte et sa mutation. Tout, dans les Lettres d'un voyageur, comme le titre significativement nous le rappelle, est dans le passage, le voyage. Les Lettres d'un voyageur, me semble-t-il, s'appréhendent exemplairement dans cet entre-deux, dans ce déjà là et ce pas tout à fait disparu.

Le recueil garde donc les marques, comme des stigmates, de sa première forme. Ces cicatrices doivent d'autant moins surprendre le lecteur que la consultation attentive de la revue prouve que les "Lettres d'un voyageur" ne constituaient pas a priori un recueil et que le travail de collecte a fabriqué une unité qui n'existait pas préalablement. On peut en effet distinguer, parmi les douze lettres finalement publiées en recueil, trois cas.

Le premier cas, le plus simple, désigne la série des «Lettres d'un voyageur " parues sous ce titre dans la revue et désignant des lettres écrites lors du voyage en Italie (soit les lettres I, II et III) : c'est, en apparence, le noyau fondateur du recueil caractérisé par la thématique du voyage et l'unité de temps. Peut-être à ce noyau pourrait-on ajouter la lettre $\mathrm{X}$ qui décrit effectivement un voyage de la Vallée Noire à la vallée de Chamonix.

Le deuxième cas rassemble un sous-ensemble des lettres effectivement parues sous le titre, "Lettres d'un voyageur", même si l'unité thématique ou discursive n'est pas évidente (il n'y a pas à proprement parler de voyage). Aux lettres I, II, III et X s'ajoutent alors dans le recueil les lettres IV, VI, VII, IX et XI. S'il s'agit d'un deuxième ensemble de lettres d'un voyageur, celui-ci est quasiment induit et suscité par la titraille de la revue et l'unité générique.

Mais à ces textes, le recueil, par un geste brutal dont le péritexte et le paratexte d'ailleurs gardent trace, agglomère trois textes plus ou moins hétérogènes aussi bien par la forme que par le fond qui sont censés devenir, par l'effet même du recueillement, des «Lettres d'un voyageur» comme les autres. Dans Histoire de ma vie, George Sand corrobore notre démonstration d'un évident manque d'homogénéité originelle du recueil :

Voici quel était ce plan, suivi au début de la série, mais dont je me suis écartée en continuant, et que je semble avoir tout à fait perdu de vue à la fin. Cet abandon apparent vient surtout de ce que j'ai réuni sous le même titre de Lettres d'un voyageur, diverses lettres ou séries qui ne rentraient pas dans l'intention et dans la manière des premières ${ }^{8}$.

8. G. Sand, Histoire de ma vie, in Euvres autobiographiques, édition de G. Lubin, op. cit., vol. II, I97I, p. 298. 
Leur insertion se fait quelquefois aux forceps et cette opération, non seulement par son résultat mais surtout par son effectuation, fonde le recueil.

Dans cette série de trois lettres figure le morceau «Le Prince» publié le Is octobre I834 que rien a priori ne paraissait destiner à devenir une lettre d'un voyageur. Sa forme dialogique elle-même l'éloigne de l'unité épistolaire du recueil (il fait partie d'une autre série encore en gestation, les dialogues politiques ${ }^{9}$ ). Sa réflexion essentiellement politique, anachronique pour une lettre d'un voyageur en 1834, contraint George Sand à une entorse chronologique lors de la fondation du recueil. Replacée derrière la lettre à Éverard et la lettre sur la maison déserte qui se termine sur une évocation à la liberté d'expression menacée, "Le Prince», malgré le décalage générique, s’homogénéise dans la continuité thématique et idéologique sans finalement susciter de rejet particulier du recueil. «Le Prince peut et doit même trouver place parmi ces morceaux, sous forme de fragment de lettre», avait écrit George Sand à Buloz le 2 août 1836.

Dans cette série, un texte encore plus intéressant est la lettre à Nisard, lettre de circonstance et non de voyageur, publiée dans la Revue de Paris en mai 1836 et dont l'agrégation semble au premier abord peu problématique. Elle prend approximativement sa place chronologique dans la liste. Le détournement de sens est cependant immense puisque l'insertion de cette lettre parachève, comme l'a montré Nicole Mozet ${ }^{\text {to }}$, et même, dirions-nous aussi, établit le traité esthétique. Elle produit un effet de clôture dont la dernière touche sera portée, en 1843 , par l'ajout de la préface.

Le dernier texte se caractérise d'emblée par une forte hétérogénéité puisqu'il appartient à un autre projet d'œuvre et à un autre projet éditorial. Parues le Is janvier I835 sous le titre «Lettres d'un oncle», les lettres réinsérées sous le numéro $\mathrm{V}$ dans le recueil constituaient un ensemble homogène et autonome salué comme tel par Liszt et Marie d'Agoult. L'énonciateur des « Lettres d'un oncle» ne ressemblait en rien au jeune voyageur des lettres précédentes. George Sand a même réuni arbitrairement deux séries parallèles : les «Lettres d'un voyageur» d'un côté, les «Lettres d'un oncle» de l'autre. Sa correspondance de l'époque témoigne de l'opération de transfert. Elle écrit à Buloz le 2 août I836 : «Je puis, si cela ne suffit, retrancher des lettres d'un oncle beaucoup de puérilités, ajouter quelque chose et donner le titre et la forme d'une lettre de voyageur». Plus étonnant encore est le devenir de cette

9. Parmi ces dialogues politiques, nous aurons par exemple les «Dialogues familiers sur la poésie des prolétaires", Revue indépendante, janvier et décembre I842, ou encore "Le Père Vatout-seul ", Almanach populaire de la France pour I845, 25 décembre I844.

Io. N. Mozet, George Sand, écrivain de romans, op. cit., p. 47-52. 
opération. Encore marginale en I836 dans l'esprit de Sand, la lettre d'un oncle devient en quelques mois matricielle et fondatrice. Ainsi dans Histoire de ma vie, George Sand, parlant des "Lettres d'un voyageur» opère une confusion volontaire et significative avec les «Lettres d'un oncle»: «Mais peu importe ce qui me fut imputé comme personnel et réel dans les Lettres d'un oncle, car c'est sous ce titre que parut d'abord le sixième numéro des Lettres d'un voyageur, et c'est sous ce titre que je m'étais promis de continuer dans la même donnée $e^{\mathrm{II}}{ }^{\prime}$. La lettre $\mathrm{V}$, la plus disparate du recueil aurait donc eu finalement une valeur matricielle. C'est paradoxalement cette opération de torsion énonciative fortuite, ce balancement constant entre lettre d'un voyageur et lettre d'un oncle qui fonde la spécificité et l'unité du recueil. Ce personnage de l'oncle réapparaît d'ailleurs dans les lettres plus tardives comme la lettre à Meyerbeer (p. 925 ; p. 302) et erre comme un fantôme dans les Nouvelles Lettres d'un voyageur.

La prise en compte du transfert de la revue vers le recueil permet donc d'insister sur la vigueur scénographique des options choisies par Sand (la fusion "Lettres d'un oncle» - «Lettres d'un voyageur») et sur la force de la forme recueil elle-même qui est une forme qui peut créer du sens et recréer de l'articulation. Le recueil va vers une refictionnalisation et une rescénarisation complète de l'ensemble, un déplacement du sens du voyage de plus en plus symbolique (on est loin du récit de voyage de la Revue des Deux Mondes) et un choix de plus en plus radical de l'autofiction contre l'autobiographie sensible dans le balancement identitaire oncle/voyageur.

La naissance des Lettres d'un voyageur est bien postérieure à la publication des premières lettres dans la revue même si le geste recréatif est volontairement gommé par Sand dans sa préface : "Je n’ai pourtant rien changé à cette œuvre informe, si ce n'est quelques mots impropres et une ou deux pages de lieux communs sans intérêt» (p. 649 ; p. 4I). Le travail opéré est puissant et notable au niveau des seuils tout d'abord : titre fédérateur à la double vertu poétique et thématique ; signature fédératrice installée sur la couverture encore rappelée à la clôture du recueil par ce syntagme plurivoque, "George Sand et compagnie» (p. 942 ; p. 318) ; apposition assez rapide d'une préface. La transformation est aussi considérable au niveau des textes : nombreuses coupes ; rétablissement global de l'ordre chronologique de la rédaction à l'encontre de l'ordre initial de la publication ; gommage de toutes les marques de la féminité et du féminin. C'est uniquement dans le recueil - et non dans la revue - que Sand construit « un lieu symbolique, hors sexe et hors société, qui permet d'être ailleurs mais pas vraiment en dehors ${ }^{12} »$.

II. G. Sand, Histoire de ma vie, édition de G. Lubin, op. cit., vol. II, p. 300.

I2. N. Mozet, George Sand, écrivain de romans, op. cit., p. 32. 
Le plus important est que le passage au recueil comme forme hyperbolise, dans les Lettres d'un voyageur, la complexité de l'instance discursive. Déjà George Sand insiste abondamment dans sa préface sur la complexité du «je»

Ainsi, en parlant tantôt comme [...] un vieux oncle podagre, tantôt comme un jeune soldat impatient, je n'ai fait autre chose que de peindre mon âme sous la forme qu'elle prenait à ces moments-là : tantôt insouciante et folâtre, tantôt morose et fatiguée, tantôt bouillante et rajeunie. [...] Je n'ai pas voulu qu'on cherchât, sous le déguisement de ce problématique voyageur, le secret d'une individualité bizarre ou remarquable. (P. 647 ; p. 38-39.)

Mais c'est bien le passage du recueil à la revue qui accentue tous ces effets problématiques. D'abord la superposition de l'énonciation d'origine des textes repris et de celle qui accompagne la nouvelle publication souligne d'emblée l'ambiguïté énonciative car le recueil suppose toujours deux actes de la part de l'écrivain : celui d'une écriture première et celui d'une (re)lecture génératrice d'un réinvestissement des textes. D'autre part, la constitution d'une figure de l'auteur-essayiste dans l'espace du recueil, suppose un «je» qui dépasse les différentes incarnations énonciatives de la première personne dans les textes. Ce «je» est bien exemplifié dans l'expression «George Sand et compagnie» qui, d'un côté, renvoie à la couverture des textes et clôt exemplairement le recueil mais, de l'autre, rappelle de manière exemplaire l'ambiguïté de l'énonciation.

Si le recueil d'articles peut, dans certains cas, tuer l'énoncé lorsque les circonstances de son énonciation première, son enracinement historique constituent son principe vital (comme dans certains recueils journalistiques très datés), ici le recueil des Lettres d'un voyageur parachève l'œuvre : il permet, avec la complicité du lecteur, d'additionner et de rendre compatibles la multiplicité des énonciations fictives imaginées par Sand ; il permet aussi de manifester exemplairement cette ambiguïté de l'énonciation et de la parole en mettant en évidence la multiple appartenance temporelle, spatiale et générique du texte et donc de l'énonciateur. La mise en recueil constitutive du projet admet et même souligne en palimpseste l'origine périodique et mutante de la parole première de la revue. La superposition de postures énonciatives caractéristique des lettres sandiennes se retrouve dans le passage de la revue au recueil. Il fallait dans ce vacillement des identités que s'opère aussi cette métamorphose générique, déjà sensible d'ailleurs dans l'utilisation publique de lettres privées. Si le voyageur, comme nous avons essayé de le démontrer ici, est le texte lui-même, rien d'étonnant donc à ce qu'il soit problématique. 